\title{
Virology, Human
}

National Cancer Institute

\section{Source}

National Cancer Institute. Virology, Human. NCI Thesaurus. Code C19119.

The study of those viruses that infect humans and the diseases they cause. 